\title{
IGR J17488-2338: a newly discovered giant radio galaxy
}

\author{
M. Molina ${ }^{1}$, L. Bassani ${ }^{1}$, A. Malizia ${ }^{1}$, A. J. Bird ${ }^{2}$, A. Bazzano ${ }^{3}$, P. Ubertini ${ }^{3}$, and T. Venturi ${ }^{4}$ \\ 1 IASF/INAF, via Gobetti 101, 40129 Bologna, Italy \\ e-mail: molina@iasfbo.inaf.it \\ 2 School of Physics and Astronomy, University of Southampton, SO17 1BJ Southampton, UK \\ 3 IAPS/INAF via Fosso del Cavaliere 100, 00133 Roma, Italy \\ ${ }^{4}$ IRA/INAF, via Gobetti 101, 40129 Bologna
}

Received 10 February 2014 / Accepted 5 March 2014

\section{ABSTRACT}

\begin{abstract}
We present the discovery of a large-scale radio structure associated with IGR J17488-2338, a source recently discovered by INTEGRAL and optically identified as a broad-line AGN at redshift 0.24. At low frequencies, the source properties are those of an intermediate-power FR II radio galaxy with a linear size of $1.4 \mathrm{Mpc}$. This new active galaxy is therefore a member of a class of objects called giant radio galaxies (GRGs), a rare type of radio galaxy with physical sizes larger than $0.7 \mathrm{Mpc}$; they represent the largest and most energetic single entities in the Universe and are useful laboratories for many astrophysical studies. Their large-scale structures could be due either to special external conditions or to uncommon internal properties of the source central engine. The AGN at the centre of IGR J17488-2338 has a black hole of $1.3 \times 10^{9}$ solar masses, a bolometric luminosity of $7 \times 10^{46} \mathrm{erg} \mathrm{s}^{-1}$ and an Eddington ratio of 0.3 , suggesting that it is powerful enough to produce the large structure observed in radio. The source is remarkable also for other properties, among which its X-ray absorption, at odds with its type 1 classification, and the presence of a strong iron line, which is a feature not often observed in radio galaxies.
\end{abstract}

Key words. gamma rays: galaxies - radio continuum: galaxies - galaxies: active

\section{Introduction}

Powerful extragalactic radio sources are galaxies (and/or quasars) hosting active galactic nuclei (AGNs), which produce jets and extended radio emitting regions (lobes) of plasma. Some of them are characterised by giant structures and are known as giant radio galaxies (GRGs), formally those with linear sizes larger than 0.7 Mpc (e.g. Lara et al. 2001; Ishwara-Chandra \& Saikia 1999, scaled for the cosmology adopted here of $H_{0}=$ $\left.71 \mathrm{~km} \mathrm{~s}^{-1} \mathrm{Mpc}^{-1}, \Omega_{\mathrm{m}}=0.27, \Omega_{\Lambda}=0.73\right)$. These objects represent the largest and most energetic single entities in the Universe and it is possible that they play a special role in the formation of large-scale structures. They generally belong to the FR II (Fanaroff \& Riley 1974) radio morphology (edge brightened), have relatively low radio power $\left(\log P_{1.4 \mathrm{GHz}(\mathrm{W} / \mathrm{Hz})} \gtrsim 24.5\right.$; Owen \& Laing 1989), and reside in elliptical galaxies and quasars. Giant radio galaxies are very useful for studying many astrophysical issues, such as understanding the evolution of radio sources, probing the intergalactic medium at different redshifts and investigating the nature of their central AGNs. There are various scenarios that try to explain this phenomenon. For example, GRGs could be very old sources that have had enough time to evolve to such large sizes. Alternatively, they could grow in an intergalactic medium whose density is smaller than that surrounding smaller radio sources, or, instead, their AGNs are extremely powerful and/or long-lived and thus able to produce such large-scale structures. Because of their large sizes and relatively low radio power, the surface brightness of GRGs is low. This is why they are so difficult to find even in radio surveys and why finding a new member of the class is interesting and useful. Here we report on the discovery and subsequent analysis of the INTEGRAL source IGR J17488-2338, which we have identified as an FR II radio galaxy with a linear size of $1.4 \mathrm{Mpc}$, thus well above the threshold for it to be a GRG. In optical terms, the galaxy is a Seyfert 1.5 at redshift 0.24 and the source is remarkable for other properties, among which its absorption characteristics, at odds with its Type 1 classification, and the presence of an iron line in the X-rays. In particular, the core of this giant radio galaxy is extremely powerful in X/gamma-rays, is highly massive, and is able to accrete very efficiently: it is possible that these extreme properties provide the necessary conditions (high jet power or long activity time) to produce the giant radio structure observed in this newly discovered radio galaxy.

\section{Source discovery and identification}

The source IGR J17488-2338 was first reported by Bird et al. (2010) as an unidentified and faint X-ray source detected by IBIS during observations of the Galactic plane. Here we use data collected in the fourth IBIS survey (Bird et al. 2010), which consists of all exposures from the beginning of the mission (November 2002) up to April 2008. The total exposure on this region is $\sim 6.2 \mathrm{Ms}$. The IBIS/ISGRI images for each available pointing were generated in various energy bands using the ISDC offline scientific analysis software version 7.0 (OSA 7.0; Goldwurm et al. 2003) and the light curves generated with this method where analysed first at revolution level and then applying the bursticity analysis. The source was clearly detected at the revolution level, but barely over the entire observation period with an upper limit on the average $20-40 \mathrm{keV}$ flux of $0.2 \mathrm{mCrab}$ and a $4 \sigma$ detection of $0.4 \pm 0.1 \mathrm{mCrab}$ in the $40-100 \mathrm{keV}$ band. The source has a bursticity of 5.3 suggesting significant variability. The peak flux, defined as the mean flux during the single period of time over which the significance is maximised (roughly 
2.6 days around October $13-15,2003)$ is $3.4 \pm 0.8 \mathrm{mCrab}$ in the $20-40 \mathrm{keV}$ band; the source dynamic range is therefore at least a factor of 17. The source spectrum corresponding to this period of high flux level is well fitted by a power law with $\Gamma=1.30 \pm 0.35$ and a $20-100 \mathrm{keV}$ flux of $1.40 \times 10^{-11} \mathrm{erg} \mathrm{cm}^{2} \mathrm{~s}^{-1}$.

The source IGR J17488-2338 is located at RA(J2000) = $17^{\mathrm{h}} 48^{\mathrm{m}} 47.3^{\mathrm{s}}$ and $\operatorname{Dec}(\mathrm{J} 2000)=-23^{\mathrm{d}} 38^{\mathrm{m}} 06.0^{\mathrm{s}}$, with a positional uncertainty of $\sim 4.7^{\prime}$. It has not yet been reported in the latest Swift/BAT surveys (Baumgartner et al. 2013; Cusumano et al. 2010); we note, however, that these surveys mainly look for persistent sources.

Stephen et al. (2010), by means of a cross-correlation analysis with the XMM Slew Survey Catalogue (Saxton et al. 2008), reported the first X-ray counterpart of IGR J174882338 in a source (XMMSL1 J174838.8-233527) located at $\operatorname{RA}(\mathrm{J} 2000)=17^{\mathrm{h}} 48^{\mathrm{m}} 38.9^{\mathrm{s}}$ and $\operatorname{Dec}(\mathrm{J} 2000)=-23^{\mathrm{d}} 35^{\mathrm{m}} 26.8^{\mathrm{s}}$ (with an error radius of 5.1") and displaying a $2-12 \mathrm{keV}$ flux of $2.5 \times 10^{-12} \mathrm{erg} \mathrm{cm}^{2} \mathrm{~s}^{-1}$. In subsequent X-ray follow-up observations with Swift/XRT, Landi et al. (2011) confirmed this association and measured the source broad-band X-ray spectrum for the first time: over the $0.3-100 \mathrm{keV}$ band, IGR J174882338 displays a power-law continuum with a flat spectral index $(\Gamma=1.3)$, absorption in excess to the Galactic value $\left(N_{\mathrm{H}}=\right.$ $1.3 \times 10^{23} \mathrm{~cm}^{-2}$, and a $2-100 \mathrm{keV}$ flux of $\sim 2 \times 10^{-11} \mathrm{erg} \mathrm{cm}^{2} \mathrm{~s}^{-1}$. Despite being classified as a variable object in the fourth IBIS catalogue (Bird et al. 2010), the cross-calibration constant between INTEGRAL/IBIS and Swift/XRT turned out to be compatible with unity; similarly, the source $2-10 \mathrm{keV}$ flux was found to be fully consistent with the XMM Slew one.

The reduction in the source positional uncertainty made possible by the identification of the X-ray counterpart allowed additional radio and optical follow-up studies. First, the X-ray source was found to coincide with the central region of a bright radio source, showing a double lobe morphology (Stephen et al. 2010); this association immediately suggested an extragalactic nature for the source, i.e. the discovery of a new radio galaxy with an active nucleus located behind the Galactic plane. IGR J174882338 was then optically classified by Masetti et al. (2013) as a Seyfert 1.5 galaxy at $z=0.24$. These authors also measured the mass of the source's central black hole using the broad-line velocities; for IGR J17488-2338 the $\mathrm{H} \beta$ emission line provides a black hole of $1.3 \times 10^{9}$ solar masses.

Figure 1 shows a cut-off image from the $1.4 \mathrm{GHz}$ Northern VLA Sky Survey (NVSS; Condon et al. 1998) of the region surrounding IGR J17488-2338; the figure shows the clear presence of a double-lobe radio source inside the INTEGRAL positional uncertainty (large white circle) with the cross marking the position of the X-ray source detected by the XMM Slew survey. The two radio lobes are fairly symmetrical, roughly ellipsoidal, and placed on either side of the compact X-ray source; they are most likely powered by two symmetrically-positioned jets emanating from the AGN core which is marked by the X-ray emission.

\section{Additional information on the source}

The X-ray counterpart of IGR J17488-2338 is listed in the WISE survey (Wright et al. 2010) with 3.5, 4.6, 11.6, and $22.1 \mu \mathrm{m}$ magnitudes of $12.12,11.02,8.5$, and 5.9 , respectively; at the source redshift the estimated WISE luminosity is of the order of $3 \times 10^{45} \mathrm{erg} \mathrm{s}^{-1}$. The first two magnitudes are reported to be variable with a high probability. The 22.1-micron WISE image of the region around the source is shown in Fig. 2 overlaid with the $1.4 \mathrm{GHz}$ contours. The WISE object is clearly associated with the core of the radio galaxy, while no extended infrared

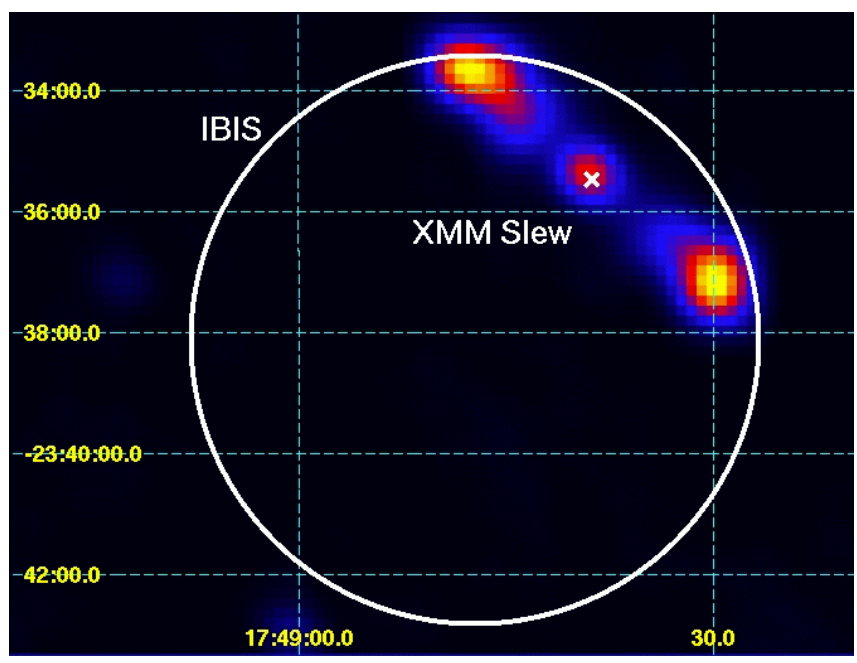

Fig. 1. 1.4 GHz NVSS image of IGR J17488-2338. The white circle corresponds to the IBIS error circle, while the white cross marks the XMM Slew position. Image courtesy of Dr R. Landi.

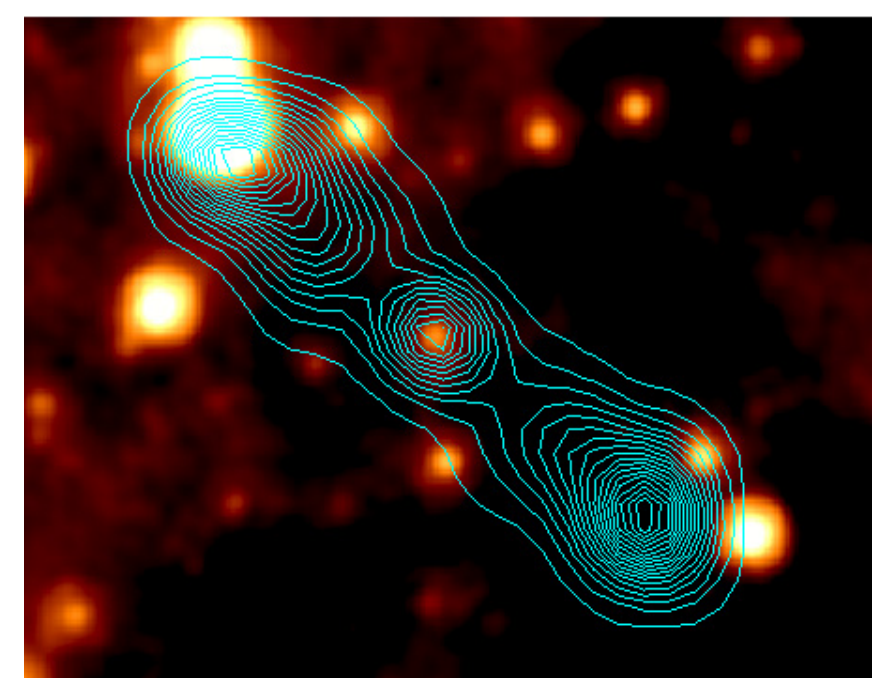

Fig. 2. 22.1-micron WISE image of the region containing IGR J174882338; NVSS radio contours are superimposed.

emission appears to be produced in correspondence of the lobes (the bright spot associated with one of them is probably a background/foreground object); this is consistent with the fact that no significant thermal emission is expected to be associated with the lobes of radio galaxies.

The radio core of IGR J17488-2338 is also reported as a 2MASS object with J, H, K fluxes ranging from 14 to $13 \mathrm{mag}$, providing a near infrared luminosity around $4 \times 10^{45} \mathrm{erg} \mathrm{s}^{-1}$. In the optical the source has $R$ and $B$ magnitudes of 17.2 and 19, respectively, yielding a lumonosity of $2 \times 10^{45} \mathrm{erg} \mathrm{s}^{-1}$. The source is quite red $(R-K=4.3)$, possibly due to heavy obscuration along the line of sight related to the source location close to the Galactic plane; indeed Masetti et al. (2013) measure 4.9 mag of extinction due to our Galaxy alone.

To improve the quality of the source X-ray spectrum, we also acquired an XMM-Newton observation that was performed on September 9, 2013, during revolution 251. The EPIC-pn image of the region shows two well detected objects: the one already seen by Swift/XRT and by the XMM Slew survey, plus an extra source located at RA $(J 2000)=17^{\mathrm{h}} 48^{\mathrm{m}} 49.49^{\mathrm{s}}$ and $\operatorname{Dec}(\mathrm{J} 2000)=-23^{\mathrm{d}} 37^{\mathrm{m}} 39.8^{\mathrm{s}}$. This second source is, however, 
very soft (undetected above a few $\mathrm{keV}$ ) and unlikely to be an alternative/valid counterpart of IGR J17488-2338: it is instead probably associated with coronal emission from the star HD 161837 of G5V spectral type. Therefore, XMM-Newton also confirms the association with the radio source and further indicates that no X-ray emission is detected from the lobes, but only from the central radio core.

The EPIC-pn (Turner et al. 2001) data were reprocessed using the XMM-Newton Standard Analysis Software (SAS) version 12.0.1 and employing the latest available calibration files. Only patterns corresponding to single and double events (PATTERN $\leq 4)$ were taken into account; the standard selection filter FLAG $=0$ was applied. The EPIC-pn nominal exposure of $25 \mathrm{ks}$ was filtered for periods of high background and the resulting exposure amounts to $\sim 14 \mathrm{ks}$. Source counts were extracted from a circular region of $23^{\prime \prime}$ radius centred on the source, while background spectra were extracted from two circular regions of $20^{\prime \prime}$ of radius close to the source. The ancillary response matrix (ARF) and the detector response matrix (RMF) were generated using the XMM-SAS tasks arfgen and rmfgen and spectral channels were rebinned in order to achieve a minimum of 20 counts per bin. No pile-up was detected for this source. The spectral analysis was performed using XSPEC v.12.8.0 (Arnaud 1996); errors are quoted at 90\% confidence level for one parameter of interest $\left(\Delta \chi^{2}=2.71\right)$. As was done previously, we combined the soft X-ray data obtained from the EPIC-pn observation to the IBIS/ISGRI points obtained during the source outburst; to take into account any mismatch between these two instruments and/or any flux variability between observations, a cross-calibration constant was introduced in the fit.

The $0.5-110 \mathrm{keV}$ broad-band spectrum was first fitted using a simple power law absorbed only by Galactic column density, which in the source direction is $N_{\mathrm{H}}=0.41 \times 10^{22} \mathrm{~cm}^{-2}$; the fit is quite poor, yielding a $\chi^{2}=575.4$ for 321 degrees of freedom (d.o.f.) and a flat power-law photon index $(\Gamma=0.61 \pm 0.04)$. In the model-to-data ratios, clear residuals are present at low energies (probably due to absorption in excess of the Galactic value) and around the iron $\mathrm{K} \alpha$ line (which in the observer's rest frame is around $5.5 \mathrm{keV}$ ). The addition of intrinsic absorption improves the fit significantly ( $\chi^{2}=313.9$ for 320 d.o.f.), but maintains some residuals below $1 \mathrm{keV}$; the resulting column density is $N_{\mathrm{H}}=0.91_{-0.14}^{+0.15} \times 10^{22} \mathrm{~cm}^{-2}$. To achieve a better fit, we substitute the fully covering cold absorber with a partially covering model. The fit further improves $\left(\chi^{2}=307.9\right.$ for 319 d.o.f.) providing a column density $N_{\mathrm{H}}=1.14_{-0.23}^{+0.26} \times 10^{22} \mathrm{~cm}^{-2}$ covering $93 \%$ of the central source. We then added a Gaussian component, with width fixed to $10 \mathrm{eV}$, to model the iron line. This model provides further improvement in the fit $\left(\chi^{2}=297.3\right.$ for 317 d.o.f.); the spectral slope is now $\Gamma=1.37 \pm 0.11$ and the iron line is found at $E_{\mathrm{K} \alpha}=6.51 \pm 0.07 \mathrm{keV}$ (source rest frame) with an equivalent width of $128_{-62}^{+61} \mathrm{eV}$. This is the best-fit model and it is displayed in Fig. 3. Adopting a more complex model like an exponentially cut-off power-law reflected from neutral material, the fit does not improve $\left(\chi^{2}=297.36\right.$ for 315 d.o.f $)$ and only a loose constraint on the reflection fraction can be obtained $(R<1.84)$. This upper limit on $R$ is, however, compatible with what is expected from the observed value of the iron line equivalent width, since $E W / R \sim 100-130 \mathrm{eV}$ (Perola et al. 2002).

The cross-calibration constant between XMM and INTEGRAL/IBIS is found to be $1.48_{-0.34}^{+0.45}$, suggesting again that the source is not strongly variable over long periods of time despite the previous INTEGRAL indication; the $2-10 \mathrm{keV}$ flux measured during the 2013 pointing is $2 \times 10^{-12} \mathrm{erg} \mathrm{cm}^{-2} \mathrm{~s}^{-1}$, again consistent with the XMM Slew and XRT data.

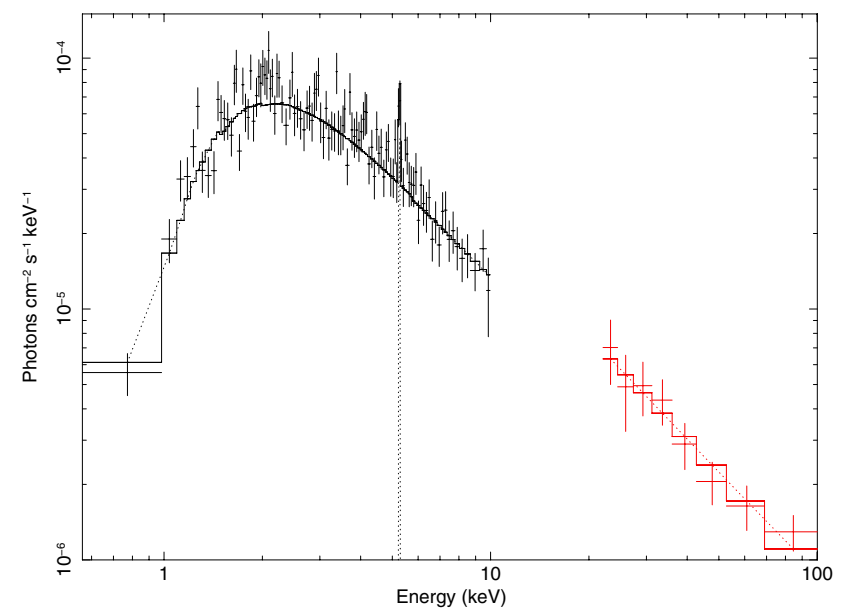

Fig. 3. $0.5-110 \mathrm{keV}$ XMM-IBIS best-fit plot; black data points are XMM measurements, while red data points are from IBIS. The model employed is a partially absorbed power law plus a neutral iron line.

The total (2-100 keV) X-ray luminosity is $2.7 \times 10^{45} \mathrm{erg} \mathrm{s}^{-1}$, confirming that the source is extremely powerful at these high energies. Taking into account the information gathered for this source in the available bands, we can estimate that the bolometric luminosity exceeds at least $10^{46} \mathrm{erg} \mathrm{s}^{-1}$. If we evaluate the bolometric luminosity, assuming the relation $\left(L_{\mathrm{Bol}}=\right.$ $25 \times L_{20-100 \mathrm{keV})}$ found by Mushotzky et al. (2008), we obtain a value of $7 \times 10^{46} \mathrm{erg} / \mathrm{s}^{1}$. The Eddington ratio is therefore around 0.3 , implying a highly efficient AGN at the centre of this galaxy.

As a final remark, we note that IGR J17488-2338 is somewhat peculiar in the X-rays. At odds with the expectations of the unified theory, it is absorbed in the X-rays, but also displays broad emission lines in the optical; furthermore, contrary to what is generally observed in radio galaxies where reprocessing features are often weak (Grandi et al. 2006), it shows a prominent iron line and could have a significant reflection component. It is clearly a source that deserves more in-depth studies to understand its peculiarities and properties at these high energies.

\section{Radio characteristics}

From the NVSS 1.4 GHz contours shown in Figs. 1 and 2, two compact regions of radio emission can be seen within the lobes. These compact regions are typical of the hotspots seen in Fanaroff-Riley Type II (FR II; Fanaroff \& Riley 1974) radio galaxies. From the NVSS image we measured the flux density of the various components by means of the AIPS tasks IMFIT (core) and TVSTAT (lobes), and obtained a flux density of $60.5 \pm 2.1 \mathrm{mJy}$ for the core, and $S_{1.4 \mathrm{GHz}}=179.8 \pm 6.3 \mathrm{mJy}$ and $157.3 \pm 5.5 \mathrm{mJy}$ for the northern and southern lobes, respectively (including the hot spots). The total flux density we measured from the image is $S_{1.4 \mathrm{GHz}}=398.5 \pm 13.9 \mathrm{mJy}$, consistent with the sum of the individual components. Unfortunately, no pointed radio observations are available in the radio archives for this radio galaxy, which prevents us even from general considerations of its properties. At $4.85 \mathrm{GHz}$, the PMN survey flux limit of $42 \mathrm{mJy}$ is used in the calculation. We should point out that the angular resolution of the PMN survey is much larger than that of NVSS (by almost a factor of 7), hence any spectral estimate

1 The Swift/BAT hard X-ray luminosity (14-195 keV) has been rescaled to the INTEGRAL/IBIS $20-100 \mathrm{keV}$ value. 
is impossible. However, from the basis of the flux density values reported here, we can safely conclude that the spectrum of the radio core is flat, pointing at ongoing nuclear activity, and that the lobes are steep, most likely as a consequence of radiative aging, which causes the frequency break in the spectrum to shift to lower frequencies (see Blundell \& Rawlings 2000).

The $1.4 \mathrm{GHz}$ total flux density of IGR J17488-2338 (398.5 mJy) corresponds to a radio power of $6.8 \times 10^{25} \mathrm{~W} \mathrm{~Hz}^{-1}$, a value well above the FRI/FRII break luminosity, and consistent with the lobe and hot spot morphology, typical of FRII radio galaxies. The core fraction derived at $1.4 \mathrm{GHz}$ from NVSS is about 0.15 . This low value is consistent with the overall low core dominance for FRII radio galaxies (e.g. Lara et al. 2001). All the above characteristics fully qualify IGR J17488-2338 as an FR II radio galaxy of intermediate power.

At the frequency of $1.4 \mathrm{GHz}$ and using a line profile across the two radio lobes, we measure the separation between the two most distant points in the lobes as 480 arcsec. At the source redshift of $z=0.24$ and assuming current cosmological parameters $\left(H_{0}=71, \Omega_{\mathrm{M}}=0.270, \Omega_{\mathrm{vac}}=0.730\right)$ the observed separation corresponds to a scale of $3.760 \mathrm{kpc} / \mathrm{arcsec}$. Therefore, IGR J17488-2338 has a projected linear extent of $1.4 \mathrm{Mpc}$, sufficiently large for it to be classified as a GRG. Despite a search for similar objects in the NVSS recently carried out by Solovyov \& Verkhodanov (2011), IGR J17488-2338 was not recognised as a giant radio galaxy, probably because of its low location on the Galactic plane; therefore, the INTEGRAL detection rediscovered this source and for the first time found that it has a giant structure.

\section{Conclusions}

We have uncovered an AGN with a large-scale radio structure in the newly discovered INTEGRAL source IGR J17488-2338. The source, which is clearly a FR II of intermediate power, has a linear size of $1.4 \mathrm{Mpc}$, which fully qualifies it as a giant radio galaxy. The source is also remarkable for other properties, among which its X-ray absorption characteristics at odds with its Type 1 classification and the presence of an iron line in the $\mathrm{X}$-rays.

It is still unclear what reasons or conditions lead to the formation of giant radio galaxies. It could be special external conditions (such as the low density of the intergalactic medium) or uncommon internal properties of the source central engine (like a high jet power or a long activity time). It is likely that none of the mentioned reasons is sufficient in itself and that several conditions must be satisfied at the same time to provide the large-scale structures seen in some radio galaxies. In the particular case of IGR J17488-2338, the properties of the central AGN are quite exceptional, suggesting that it may be capable of producing a highly powerful jet or of maintaining activity over a long period of time; either possibility provides the conditions to form a large-scale radio structure. The source is extremely bright in the X/gamma-rays: among a set of 25 radio galaxies detected so far by INTEGRAL, this is the brightest object in the sample and also one of the most efficient accretors (Molina et al., in prep.). Like Cygnus A and 4C 74.26, also included in the INTEGRAL sample of radio galaxies, IGR J17488-2338 hosts a black hole with a mass greater than $10^{9}$ solar masses; coupled with the source extension, this value perfectly fits with the linear relation found by Kuźmicz \& Jamrozy (2013) for GRG and is based on the observed linear extensions and the black hole masses derived from the $\mathrm{H} \alpha$ emission line. It is interesting to note that $4 \mathrm{C} 74.26$ is also a giant radio galaxy (Ishwara-Chandra \& Saikia 1999) with an extension of $1.9 \mathrm{Mpc}$, i.e. very similar to that of IGR J174882338. It is therefore possible that the hard X-ray selection made available by INTEGRAL and /or Swift/BAT allows the detection of the brightest AGN in the sky, and consequently also of the most powerful radio galaxies, i.e. those that are able to produce large-scale radio structures. Among the sample of INTEGRAL detected radio galaxies, six (or 24\%) qualify as giant radio galaxies; this fraction is higher than that is generally found using radio surveys, which report fractions in the range 6-11\%, depending on the survey used (Laing et al. 1983; Saripalli et al. 2012). This suggests that hard X-ray observations can provide a much more efficient way to find giant radio galaxies than radio observations, at least in the local Universe. A complete analysis of INTEGRAL radio galaxies is underway and the results regarding this issue will be presented in a forthcoming dedicated paper.

Acknowledgements. We acknowledge the Italian Space Agency (ASI) financial programmatic support via contract ASI/INAF No. 2013-025. R.O. This research has made use of the HEASARC archive provided by NASA's Goddard Space Flight Center Italian Space. We thank Dr. R. Landi for providing Fig. 1.

\section{References}

Arnaud, K. A. 1996, in Astronomical Data Analysis Software and Systems V, eds. G. H. Jacoby, \& J. Barnes, ASP Conf. Ser., 101, 17

Baumgartner, W. H., Tueller, J., Markwardt, C. B., et al. 2013, ApJS, 207, 19

Bird, A. J., Bazzano, A., Bassani, L., et al. 2010, ApJS, 186, 1

Blundell, K. M., \& Rawlings, S. 2000, AJ, 119, 1111

Condon, J. J., Cotton, W. D., Greisen, E. W., et al. 1998, AJ, 115, 1693

Cusumano, G., La Parola, V., Segreto, A., et al. 2010, A\&A, 524, A64

Fanaroff, B. L., \& Riley, J. M. 1974, MNRAS, 167, 31

Goldwurm, A., David, P., Foschini, L., et al. 2003, A\&A, 411, L223

Grandi, P., Malaguti, G., \& Fiocchi, M. 2006, ApJ, 642, 113

Ishwara-Chandra, C. H., \& Saikia, D. J. 1999, MNRAS, 309, 100

Kuźmicz, A., \& Jamrozy, M. 2013, Adv. Astron. Space Phys., 3, 42

Laing, R. A., Riley, J. M., \& Longair, M. S. 1983, MNRAS, 204, 151

Landi, R., Bassani, L., Malizia, A., et al. 2011, in Proc. Extreme and Variable High Energy Sky (Extremesky), id. 9, available at http://pos.sissa.it/ cgi-bin/reader/conf. cgi?confid=147

Lara, L., Cotton, W. D., Feretti, L., et al. 2001, A\&A, 370, 409

Masetti, N., Parisi, P., Palazzi, E., et al. 2013, A\&A, 556, A120

Mushotzky, R. F., Winter, L. M., McIntosh, D. H., \& Tueller, J. 2008, ApJ, 684, L65

Owen, F. N., \& Laing, R. A. 1989, MNRAS, 238, 357

Perola, G. C., Matt, G., Cappi, M., et al. 2002, A\&A, 389, 802

Saripalli, L., Subrahmanyan, R., Thorat, K., et al. 2012, ApJS, 199, 27

Saxton, R. D., Read, A. M., Esquej, P., et al. 2008, A\&A, 480, 611

Solovyov, D. I., \& Verkhodanov, O. V. 2011, Astrophys. Bull., 66, 416

Stephen, J. B., Bassani, L., Landi, R., et al. 2010, MNRAS, 408, 422

Turner, M. J. L., Reeves, J. N., Ponman, T. J., et al. 2001, A\&A, 365, L110

Wright, E. L., Eisenhardt, P. R. M., Mainzer, A. K., et al. 2010, AJ, 140, 1868 